\title{
A consensus-based robust secondary voltage and frequency control scheme for islanded microgrids
}

DOI:

10.1016/j.jjepes.2019.105575

\section{Document Version}

Accepted author manuscript

Link to publication record in Manchester Research Explorer

\section{Citation for published version (APA):}

Hu, J., \& Bhowmick, P. (2020). A consensus-based robust secondary voltage and frequency control scheme for islanded microgrids. International Journal of Electrical Power and Energy Systems, 116, [105575].

https://doi.org/10.1016/j.ijepes.2019.105575

\section{Published in:}

International Journal of Electrical Power and Energy Systems

\section{Citing this paper}

Please note that where the full-text provided on Manchester Research Explorer is the Author Accepted Manuscript or Proof version this may differ from the final Published version. If citing, it is advised that you check and use the publisher's definitive version.

\section{General rights}

Copyright and moral rights for the publications made accessible in the Research Explorer are retained by the authors and/or other copyright owners and it is a condition of accessing publications that users recognise and abide by the legal requirements associated with these rights.

\section{Takedown policy}

If you believe that this document breaches copyright please refer to the University of Manchester's Takedown Procedures [http://man.ac.uk/04Y6Bo] or contact uml.scholarlycommunications@manchester.ac.uk providing relevant details, so we can investigate your claim.

\section{OPEN ACCESS}




\title{
A Consensus-based Robust Secondary Voltage and Frequency Control Scheme for Islanded Microgrids
}

\author{
Junyan $\mathrm{Hu}$, Parijat Bhowmick* \\ Department of Electrical and Electronic Engineering, School of Engineering, University of Manchester, Manchester M13 9PL, U.K.
}

\begin{abstract}
This paper deals with the problem of designing a fully-distributed and robust secondary control scheme for voltage and frequency restoration of islanded microgrids along with real power sharing. Compared to the existing developments in this topic, the proposed multi-agent consensus-based secondary control scheme takes into account the effects of model uncertainty, parameter variation and unmodelled dynamics and thereby, offers a certain degree of robustness against all these factors. Due to virtue of the fully-distributed control configuration, each distributed generating (DG) unit in a microgrid requires only the information of its own and some of its neighbours but not of all other DG units attached to that microgrid. This helps in reducing the bandwidth requirement and cost of the underlying communication network and also causes enhanced flexibility and reliability of the overall microgrid operation. The proposed scheme counteracts any fluctuation in the terminal voltage and frequency of the DG units and also facilitates real power sharing among them at a pre-specified ratio despite the time-varying load disturbances, various power line faults, communication delays, etc. Finally, an exhaustive case study on a prototype IEEE 14-bus microgrid system containing five DG units has been accomplished to demonstrate the usefulness of the developed scheme.
\end{abstract}

Keywords: Secondary control, microgrid, feedback linearization, distributed cooperative control, multi-agent systems, active power sharing.

\section{Introduction}

Microgrid can be considered as the basic unit of a smart grid which integrates a number of distributed generating (DG) units and facilitates power supply to local loads and often to main grid as well [1, 2]. When a microgrid is connected to the main power grid, it is said to be working in 'grid-connected mode' and it operates in 'islanded mode' if it is isolated from the main grid [2]. Usually microgrids remain connected to the main grid, but often due to external disturbances, power line faults, unplanned outage, scheduled maintenance, etc., microgrids may loose connection to the main grid. When this happens, the primary control is brought into action to prevent any deviation in the terminal voltage and frequency of each DG unit of that isolated microgrid from the reference (or nominal) voltage $\left(V_{r e f}\right)$ and frequency $\left(\omega_{\text {ref }}\right)$ [3]. Due to the drooping characteristics of conventional primary voltage and frequency controllers, the variations in the voltage and frequency of a DG unit cannot be fully rectified which severely deteriorates the power quality and, in turn, disturbs the healthy operation of a microgrid. This necessitates the application of 'secondary control' in addition to the primary control during the islanded operating mode to accurately maintain the voltage and frequency of each DG units to the standard values $V_{\text {ref }}$ and $\omega_{\text {ref }}$. Pioneering research

\footnotetext{
*Corresponding Author. Telephone: +44-7884571252

Email addresses: Junyan.Hu@manchester . ac .uk (Junyan Hu), Parijat.Bhowmick@manchester.ac.uk (Parijat Bhowmick)
}

on the modelling, small-signal stability analysis and testing of autonomous operation of inverter-based microgrids has been reported in [4]. Varieties of control techniques have been investigated during the past few years to improve the quality and reliability of the microgrid systems. Before the advent of distributed cooperative control strategies, microgrid control schemes used to resort on centralized control techniques. But in a centralized control, each of the nodes (here the DG units) stays bidirectionally [5] connected to all the remaining modes and hence, centralized control requires a complex and expensive communication network. Moreover, a centralized control scheme ceases to work even if a single unit breaks down or loses connection to the rest of the network due to communication link failure. On the other hand, the primary advantage of a distributed control scheme is that a node is not required to communicate with all other nodes in the network. Because of this feature, distributed cooperative control techniques have been quite popular in microgrid and smart grid applications for designing controllers [6]. In [7], a dynamic optimization-based coordinated control scheme has been proposed for DC microgrids, whereas in [8], a multi-function model reference adaptive PID control law is developed to augment voltage and current control of islanded microgrids. In [9], the authors have designed a consensusbased information synchronization and distributed model predictive control scheme to ensure satisfactory voltage regulation of smart distributed networks.

Due to remarkable progress in the graph theory-based multiagent cooperative control techniques over the past two decades 
[10, 11, 12, 13], distributed cooperative secondary control of islanded microgrid operation has drawn profound attention of the smart grid control communities. In [14], a cooperative secondary frequency control scheme is developed for distributed microgrids. Moreover, [14] has also addressed fundamental issues related to data communication and network topology, which occurs during practical implementation of a distributed control scheme. In [15, 16], a distributed secondary voltage controller (SVC) has been developed for microgrids based on the small-signal dynamic model but it does not guarantee global stability due to considering incomplete plant model during the controller design. In [17], distributed cooperative control strategy is applied to design a secondary voltage control scheme and feedback linearization tool has been utilized to linearize the nonlinear plant model of a DG unit, but did not include the dynamics of the primary controller, which may deteriorate both stability and performance of the microgrid as a whole. In the sequel, the same group of authors introduced a multi-agent consensus-based distributed cooperative control framework in [18] for secondary voltage and frequency control (SVFC) of inverter-based microgrids. However, in both [17, 18], the nonzero minimum eigenvalue of the graph Laplacian matrix is directly involved in the proposed control law, which indicates that the control schemes are not fully-distributed. In order to circumvent these issues, the notion of distributed adaptive (commonly called 'fully-distributed') cooperative control has recently been introduced in the literature [3, 18, 19] for secondary controller design of islanded microgrids. The work of [19] is a major contribution in the area of secondary voltage and frequency control of isolated microgrids, however, [19] does not address the robustness issues such as model uncertainties and unmodelled dynamics. In contrast to [3, 18, 19], an alternative secondary voltage and frequency control framework has been proposed in [20] and [21] respectively for islanded microgrid operation without applying the feedback linearization technique. The notion of distributed-averaging proportionalintegral (DAPI) controllers are introduced and applied for secondary voltage and frequency control of inverter-based microgrids in [20] and [21]. Of late, in [22], the authors have designed a distributed secondary controller for islanded microgrids using the principle of optimal static output feedback control [23] based on the voltage and frequency drooping strategies used in [21]. Notable research has been conducted in [24] to develop a distributed secondary control scheme for droopcontrolled microgrids including reactive power sharing. [24] has also provided experimental results to validate their proposed theory. Leading research is carried out in [25] on designing hierarchical control framework for droop-controlled AC and DC microgrids based on ISA-95 standard that includes primary, secondary and tertiary control objectives. Later on, in [26], an extensive survey on the decentralized, distributed and hierarchical control of droop-controlled islanded microgrids has been published. In [27], signification research contribution is laid in investigating the impact of communication time delay in the secondary frequency control of an islanded microgrid with multiple DG units. Followed by [27], in [28], small-signal stability analysis of a distributed secondary frequency control scheme is performed for an islanded microgrid with multiple inverters in presence of time delay. In [29], the authors have designed a distributed secondary voltage and frequency restoration scheme for inverter-based microgrids when the communication network suffers from uncertain links.

The existing developments and the limitations therein motivate us to design a multi-agent consensus-based fullydistributed secondary control scheme to synchronise the voltage and frequency of individual DG units in a microgrid to the nominal (or reference) level during the islanded mode of operation. The contributions of this paper can be summarised as follow:

- A fully-distributed and robust SVFC framework is proposed for isolated (or islanded) microgrids which also maintains active and reactive power sharing among the DG units at a desired proportion. The scheme is developed based on a complete first-principle nonlinear dynamic model of the DGs, which may not be all identical.

- Input-output feedback linearization technique has been applied to obtain a simplified linearized model common for all DGs and to suppress the apparent structural heterogeneity present in the DG units attached to a microgrid.

- The proposed SVFC scheme being fully-distributed requires the constituent DG units to access only the local information, i.e., information of each DG unit and its neighbours, but not about the whole network. Due to virtue of this property, the SVFC scheme reduces the bandwidth requirement and also offers greater reliability and flexibility, in contrast to most of the existing approaches.

- In the proposed methodology, apart from considering the impact of bounded external disturbances (e.g., load fluctuations), an additional exogenous input term is considered to compensate for the effects due to parameter variation, model uncertainty and unmodelled dynamics.

After the theoretical development, a detailed case study on a prototype IEEE 14-bus microgrid system containing five DG units has been carried out to highlight the effectiveness and feasibility of the proposed secondary voltage and current control (SVFC) scheme. Apart from showing the nominal performance, the case study has also examined the robustness properties of the SVFC scheme to handle frequent graph topology switching, load fluctuations, plug-and-play operation and communication delay.

Rest of the paper proceeds as follow. Preliminaries on algebraic graph theory is presented in Section 2. A complete nonlinear dynamical model of an inverter-based DG unit is provided in Section 3. Section 4 contains the main contributions of the paper which develops a fully-distributed and robust secondary control scheme for voltage and frequency restoration of islanded microgrids along with maintaining active and reactive power sharing among the neighbouring DG units. In Section 5 , an in-depth case study on a prototype IEEE 14-bus microgrid 
system is considered to show usefulness of the proposed secondary control scheme. Section 6 concludes the paper mentioning future research directions.

\section{Notations}

Notations and acronyms are standard throughout. $\mathbb{R}_{>0}$ and $\mathbb{R}_{\geq 0}$ denote respectively the sets of all positive and all nonnegative real numbers. Let $I_{n}$ denote the identity matrix of dimension $n \times n$ and $\mathbf{1}_{N}$ be the vector with $N$ number of entries all being $1 . \operatorname{diag}\left\{a_{i}\right\}$ represents a diagonal matrix with the diagonal entries $a_{i} \forall i$. For a real-valued function $g(t):[0, \infty) \rightarrow$ $\mathbb{R}^{n}, g(t) \in \mathcal{L}_{2}$ if $\sqrt{\int_{0}^{\infty} g(t)^{T} g(t) \mathrm{d} t}<\infty$ and $g(t) \in \mathcal{L}_{\infty}$ if $\sup \|g(t)\|<\infty . \lambda_{\min }(X)$ denotes the minimum eigenvalue of $t \geq 0$ a matrix $X \in \mathbb{R}^{n \times n}$ that has only real eigenvalues. The Kronecker product of two matrices $A$ and $B$ is denoted by $A \otimes B$. $\|$.$\| expresses the 2-norm of a vector or a matrix. A square$ matrix $A \in \mathbb{R}^{n \times n}$ is called a non-singular $M$-matrix if all its off-diagonal elements are non-positive and all eigenvalues of $A$ have positive real parts [30].

\section{Preliminaries on graph theory}

A microgrid can be conceptualised as a multi-agent cooperative system, in which the DG units are viewed as nodes or agents and the communication links play the roles of edges. Each DG unit communicates with other DGs through a sparse communication network. Let $\mathcal{G}=(\mathcal{V}, \mathcal{E}, \mathcal{A})$ be a weighted and directed graph for which $\mathcal{V}=\{1,2, \ldots, N\}$ is a nonempty set of nodes, $\mathcal{E} \subset \mathcal{V} \times \mathcal{V}$ is the set of edges and $\mathcal{A}=\left[a_{i j}\right] \in \mathbb{R}^{N \times N}$ is the adjacency matrix corresponding to the graph. An edge denoted by $(i, j)$ indicates that information flows from node $i^{\text {th }}$ to node $j^{\text {th }}$ and, in this case, the $i^{\text {th }}$ node is considered as a 'neighbour' of the $j^{\text {th }}$ node. $a_{i j}$ is the weight of edge $(j, i)$ and $a_{i j} \neq 0$ if $(j, i) \in \mathcal{E}$. In-degree matrix associated with $\mathcal{G}$ is defined as $D=\operatorname{diag}\left\{d_{i}\right\} \in \mathbb{R}^{N \times N}$ with $d_{i}=\sum_{j=1}^{N} a_{i j}$ and the Laplacian matrix $L \in \mathbb{R}^{N \times N}$ of $\mathcal{G}$ is given by $L=D-\mathcal{A}$. Within a directed graph, a spanning tree exists if the graph has a 'root' node having directed path to each of the remaining nodes. If the $i^{\text {th }}$ node for any $i \in\{1,2, \ldots, N\}$ is attached to the root (labelled as 'node 0 '), then the node is called a 'pinned' node and an edge $(0, i)$ is said to exist between them having the weight $g_{i}>0$. We denote the pinning matrix as $G=\operatorname{diag}\left\{g_{i}\right\} \in \mathbb{R}_{\geq 0}^{N \times N}$.

The following lemma related to the properties of graph Laplacian matrix will be invoked in deriving the main results of the paper in Section 4

Lemma 1. [11] Assume there exists a spanning tree in the graph $\mathcal{G}$ and $L \in \mathbb{R}^{N \times N}$ be the corresponding Laplacian matrix. Then, $\lambda_{1}[L]=0$ is a non-repeated eigenvalue and $v_{1}=\mathbf{1}_{N}$ be the corresponding eigenvector; while $\mathfrak{R}\left\{\lambda_{i}[L]\right\} \geq 0$ for all $i \in\{2,3, \ldots, N\}$.

Note, the Laplacian matrix $L$ enjoys symmetry when $\mathcal{G}$ is undirected and in that case, the condition $\mathfrak{R}\left\{\lambda_{i}[L]\right\} \geq 0$ reduces to $\lambda_{i}[L] \geq 0 \forall i$. Lemma 2 describes an important property of $M$ matrix and it is widely applied in designing cooperative control

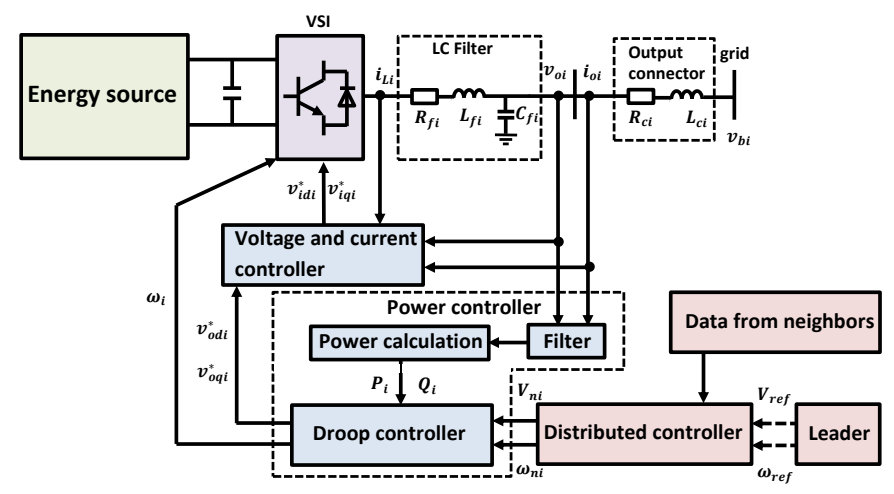

Figure 1: Schematic diagram of an inverter-based DG unit.

protocols where the graph Laplacian matrix satisfies the properties of an $M$-matrix.

Lemma 2. [31] Consider a non-singular M-matrix U. Then, there exists a positive definite matrix $\Xi=\operatorname{diag}\left\{\psi_{1}, \ldots, \psi_{N}\right\}$ such that $\Xi U+U^{T} \Xi>0$.

Let us now recall a corollary on the well-known Barbalat Lemma.

Lemma 3. [32] For some $p \in[1, \infty)$, if $g(t) \in \mathbb{L}_{p} \cap \mathbb{L}_{\infty}$ and $\dot{g}(t) \in \mathbb{L}_{\infty}$, then it holds that $\lim _{t \rightarrow \infty} g(t)=0$.

\section{Complete mathematical modelling of inverter-based DGs including primary control}

The functional block diagram of a voltage-controlled voltage-source inverter-based DG unit is depicted in Fig. 1 A microgrid consists of a number of such DG units, which are synchronised to maintain a common (usually the reference) voltage $\left(V_{r e f}\right)$ and frequency $\left(\omega_{\text {ref }}\right)$. As discussed in the Introduction, primary controller alone is not strong enough to nullify the deviations in the voltage and frequency of the individual DG units when operated in the islanded mode. Therefore, secondary control is essential in islanded mode of operation to restore the voltage $\left(v_{o i}\right)$ and frequency $\left(\omega_{i}\right)$ of each DG unit to the nominal level. Below, we provide the complete nonlinear dynamical model of an inverter-based DG unit equipped with the primary controller as derived in [1, 17, 19, 4].

Let $\delta_{i}$ denote the angle of the $i^{\text {th }}$ DG reference frame with respect to a common reference frame and satisfies the relation

$$
\dot{\delta}=\omega_{i}-\omega_{\text {com }},
$$

where $\omega_{i}$ is the angular frequency of rotation of the $i^{\text {th }}$ DG while $\omega_{\text {com }}$ represents the same associated with the common reference frame. The voltage and frequency droop characteristics exerted by the primary controller are governed by the following equations:

$$
\left\{\begin{aligned}
\omega_{i} & =\omega_{n i}-m_{p i} P_{i}, \\
v_{\text {odi }}^{*} & =V_{n i}-n_{Q i} Q_{i}, \\
v_{\text {oqi }}^{*} & =0,
\end{aligned}\right.
$$


where $m_{P i}$ and $n_{Q i}$ are the droop coefficients, selection of which depends on the active and reactive power ratings of each DG; $\omega_{i}$ is the angular frequency of $i^{\text {th }}$ DG unit fixed by the primary control; $P_{i}$ and $Q_{i}$ denote respectively the active power (in $\mathrm{kW}$ ) and reactive power (in kVAr) measured at the terminals of $i^{\text {th }} \mathrm{DG}$; $V_{n i}$ and $\omega_{n i}$ act as the reference signals to the primary controller [17, 4]. The power controller is characterised by the following differential equations:

$$
\left\{\begin{array}{l}
\dot{P}_{i}=-\omega_{c i} P_{i}+\omega_{c i}\left(v_{\text {odi }} i_{\text {odi }}+v_{\text {oqi }} i_{\text {oqi }}\right) \\
\dot{Q}_{i}=-\omega_{c i} Q_{i}+\omega_{c i}\left(v_{\text {odi }} i_{\text {oq } i}-v_{\text {oq }} i_{\text {od }}\right)
\end{array}\right.
$$

where $\omega_{c i}$ is the cutoff frequency of the low-pass filters used in the power controller block; $v_{\text {odi }}, v_{\text {oq }}, i_{\text {odi }}$ and $i_{\text {oqi }}$ are the direct and quadrature components of $v_{o i}$ and $i_{o i}$ respectively (see Fig. 1). The differential-algebraic equations of the voltage controller is given as:

$$
\left\{\begin{array}{l}
\dot{\phi}_{d i}=v_{o d i}^{*}-v_{o d i}, \\
\dot{\phi}_{q i}=v_{o q i}^{*}-v_{o q i}, \\
i_{l d i}^{*}=F_{i} i_{o d i}-\omega_{b} C_{f i} v_{o q i}+K_{P v i}\left(v_{o d i}^{*}-v_{o d i}\right)+K_{I v i} \phi_{d i}, \\
i_{l q i}^{*}=F_{i} i_{o q i}+\omega_{b} C_{f i} v_{o d i}+K_{P v i}\left(v_{o q i}^{*}-v_{o q i}\right)+K_{I v i} \phi_{q i},
\end{array}\right.
$$

where $\phi_{d i}$ and $\phi_{q i}$ are the auxiliary state variables associated with the PI controllers used in voltage control and $\omega_{b}$ denotes the nominal angular frequency. Similarly, the dynamics of the current controller is obtained as:

$$
\left\{\begin{array}{l}
\dot{\gamma}_{d i}=i_{l d i}^{*}-i_{l d i} \\
\dot{\gamma}_{q i}=i_{l q i}^{*}-i_{l q i} \\
v_{i d i}^{*}=-\omega_{b} L_{f i} i_{l q i}+K_{P c i}\left(i_{l d i}^{*}-i_{l d i}\right)+K_{I c i} \gamma_{d i} \\
v_{i q i}^{*}=\omega_{b} L_{f i} i_{l d i}+K_{P c i}\left(i_{l q i}^{*}-i_{l q i}\right)+K_{I c i} \gamma_{q i}
\end{array}\right.
$$

where $\gamma_{d i}$ and $\gamma_{q i}$ are the auxiliary states related to the PI controllers involved in current control. Finally, the governing equations of the output LC filter and output connector, as shown in Fig. 1, are mentioned below as:

$$
\left\{\begin{array}{l}
\dot{i}_{l d i}=-\frac{R_{f i}}{L_{f i}} i_{l d i}+\omega_{i} i_{l q i}+\frac{1}{L_{f i}} v_{i d i}-\frac{1}{L_{f i}} v_{o d i}, \\
\dot{i}_{l q i}=-\frac{R_{f i}}{L_{f i}} i_{l q i}-\omega_{i} i_{l d i}+\frac{1}{L_{f i}} v_{i q i}-\frac{1}{L_{f i}} v_{o q i}, \\
\dot{v}_{o d i}=\omega_{i} v_{o q i}+\frac{1}{C_{f i}} i_{l d i}-\frac{1}{C_{f i}} i_{o d i}, \\
\dot{v}_{o q i}=-\omega_{i} v_{o d i}+\frac{1}{C_{f i}} i_{l q i}-\frac{1}{C_{f i}} i_{o q i}, \\
\dot{i}_{o d i}=-\frac{R_{c i}}{L_{c i}} i_{o d i}+\omega_{i} i_{o q i}+\frac{1}{L_{c i}} v_{o d i}-\frac{1}{L_{c i}} v_{b d i}, \\
\dot{i}_{o q i}=-\frac{R_{c i}}{L_{c i}} i_{o q i}-\omega_{i} i_{o d i}+\frac{1}{L_{c i}} v_{o q i}-\frac{1}{L_{c i}} v_{b q i},
\end{array}\right.
$$

where $v_{b d i}$ and $v_{b q i}$ are the direct and quadrature components of the microgrid bus voltage $v_{b}$ as shown in Fig. 11 Now, the above set of equations (1)-(6) can be expressed in a compact form describing the input affine nonlinear state-space model of the $i^{\text {th }}$ DG unit:

$$
\begin{aligned}
\dot{x}_{i} & =f_{i}\left(x_{i}\right)+g_{i}\left(x_{i}\right) u_{i}+k_{i}\left(x_{i}\right) D_{i}, \\
y_{1 i} & =v_{o d i}=h_{1 i}\left(x_{i}\right), \\
y_{2 i} & =\omega_{i}=\omega_{n i}-m_{p i} P_{i}=h_{2 i}\left(x_{i}\right)+e_{i} u_{i},
\end{aligned}
$$

where the state vector is given by $x_{i}=\left[\delta_{i}, P_{i}, Q_{i}, \phi_{d i}, \phi_{q i}, \gamma_{d i}\right.$, $\left.\gamma_{q i}, i_{l d i}, i_{\text {lqi }}, v_{\text {odi }}, v_{\text {oqi }}, i_{\text {odi }}, i_{\text {oqi }}\right]^{T} ; y_{i}=\left[y_{1 i}, y_{2 i}\right]^{T}=\left[v_{\text {odi }}, \omega_{i}\right]^{T} \mathrm{de}-$ notes the output; $u_{i}=\left[u_{1 i}, u_{2 i}\right]^{T}=\left[V_{n i}, \omega_{n i}\right]^{T}$ represents the control input; and $D_{i}=\left[\omega_{c o m}, v_{b d i}, v_{b q i}\right]^{T}$ symbolizes the known disturbance input.

Remark 1. Presently, the paper deals with the control aspects of the microgrid and hence, the paper has not explicitly considered the issues related with the input energy sources feeding the inverter unit. It is assumed that a high-valued capacitor (or solid-state voltage clamping unit) is used to maintain the voltage input to the inverter within allowable range. Theoretically, any fluctuation in the voltage input to the inverter causes a drift in the output line current $i_{L_{i}}[33]$, which is then fed back to the voltage and current controller block to take necessary corrective action to prevent this variation by manipulating the voltage reference $v_{i}^{*}$ to the inverter as shown in Fig. 1. Furthermore, the effect of any disturbance in the input voltage due to stochastic nature of the external energy sources is considered during the controller design via including the known disturbance input $D_{i}$ in (7a) and an additional external disturbance input $d_{i}$ (will be introduced in Section 4.1).

\section{Development of a fully-distributed and robust secondary control scheme for islanded microgrid operation}

The primary objective of secondary voltage and frequency control (SVFC) is to generate appropriate reference signals $V_{n i}$ and $\omega_{n i}$ for the primary controller such that the output voltage magnitude $\left(v_{o, \text { magi }}\right)$ and frequency $\left(\omega_{i}\right)$ of each DG unit can be reinstated to the nominal values, i.e., $V_{\text {ref }}$ and $\omega_{\text {ref }}$, along with facilitating active and reactive power sharing among all the DG units connected to a microgrid at a pre-specified ratio.

\subsection{Distributed and robust secondary voltage control}

In this subsection, a fully-distributed and robust secondary voltage control (SVC) protocol has been introduced that assists the DG units to synchronise with the nominal grid voltage during the islanded mode of operation. As the $d$ and $q$ components lie in quadrature, the output voltage magnitude of the $i^{\text {th }} \mathrm{DG}$ unit is given by

$$
v_{o, \text { magi }}=\sqrt{v_{o d i}^{2}+v_{o q i}^{2}} \quad \forall i \in\{1,2, \ldots, N\} .
$$

It is already discussed that the primary voltage control strategy makes $v_{o q i}=0$ and hence, synchronisation of $v_{o, \text { magi }}$ to $V_{\text {ref }}$ is equivalent to synchronisation of $v_{\text {odi }}$ to $V_{\text {ref }}$ for each $i$. Therefore, secondary voltage control requires to find an appropriate control input $u_{i}$ (i.e., $V_{n i}$ ) such that $v_{\text {odi }}$ asymptotically follows 
$V_{\text {ref }}$ for each $i$. As the dynamics of DG units described via (7a)(7c) are nonlinear and coupled, we apply input-output feedback linearization technique to transform the nonlinear dynamics into the 'normal' form (also known as Brunovsky canonical form, see [32] for details) for designing an SVC scheme. The direct relationship between $y_{1 i}$ and $u_{1 i}$ is obtained as

$$
\ddot{y}_{1 i}=L_{F i}^{2} h_{1 i}+L_{g i} L_{F i} h_{1 i} u_{1 i},
$$

where $L_{F_{i}} h_{1 i}=\left(\frac{\partial h_{1 i}}{\partial x_{i}}\right) F_{i}$ is defined as the Lie derivative of $h_{1 i}$ with respect to

$$
F_{i}\left(x_{i}\right)=f_{i}\left(x_{i}\right)+k_{i}\left(x_{i}\right) D_{i} .
$$

Similarly the higher-order Lie derivative operators are defined as $L_{F_{i}}^{2} h_{1 i}=L_{F_{i}}\left(L_{F_{i}} h_{1 i}\right)$ and $L_{g_{i}} L_{F_{i}} h_{1 i}=\left(\frac{\partial\left(L_{F_{i}} h_{1 i}\right)}{\partial x_{i}}\right) g_{i}$. The auxiliary control law $v_{i}$ is now selected as

$$
v_{i}=L_{F i}^{2} h_{1 i}+L_{g i} L_{F i} h_{1 i} u_{1 i}+d_{i}, \quad \forall i
$$

where $d_{i}$ is an unknown input considered additionally to account for the external disturbances. Furthermore, $d_{i}=W_{u d} \bar{d}_{i}$ can be chosen where the matrix $W_{u d}$ is used to model the effects of plant uncertainties and unmodelled dynamics. Equations (9) and (11) together yield a second-order LTI sub system

$$
\ddot{y}_{1 i}=v_{i}+d_{i} \quad \forall i .
$$

The control input $u_{1 i}$ can be expressed in terms of $v_{i}$ as

$$
u_{1 i}=\left(L_{g i} L_{F i} h_{1 i}\right)^{-1}\left(-L_{F i}^{2} h_{1 i}+v_{i}\right) \quad \forall i .
$$

The LTI subsystem (12) can be expressed in a state-space form

$$
\dot{z}_{i}=A z_{i}+B v_{i}+w_{i} \quad \forall i
$$

where $z_{i}=\left[\begin{array}{cc}y_{1 i} & \dot{y}_{1 i}\end{array}\right]^{T}=\left[\begin{array}{cc}v_{\text {odi }} & \dot{v}_{\text {odi }}\end{array}\right]^{T}, A=\left[\begin{array}{cc}0 & 1 \\ 0 & 0\end{array}\right], B=$ $\left[\begin{array}{ll}0 & 1\end{array}\right]^{T}$ and $w_{i}=\left[\begin{array}{ll}0 & d_{i}\end{array}\right]^{T}$. Thus, applying input-output feedback linearization, (7a)-(7b) has been transformed into (14) and a set of internal dynamics. Note, the pair $(A, B)$ is always controllable. Now, the reference generator is described by

$$
\dot{z}_{0}=A z_{0}
$$

where $z_{0}=\left[\begin{array}{ll}V_{\text {ref }} & \dot{v}_{r e f}\end{array}\right]^{T}$. Also note that since $V_{\text {ref }}$ is constant, $\dot{z}_{0}=0$. Below, we declare several assumptions which are prerequisite for deriving the SVC protocol (i.e., Theorem 1).

Assumption 1. The topology among N DG units is undirected and connected. There exists a spanning tree in the communication graph $\mathcal{G}$ for which the root node (labelled as ' $O$ ') is the reference generator and $g_{i}>0$ for at least one $D G$ unit. In this case, $L+G>0$ [5] 18]. Note $L+G$ is also a non-singular M-matrix.

Assumption 2. The external disturbances are bounded, i.e., there exists a set of constants $\gamma_{i}$ such that $\left\|d_{i}\right\| \leq \gamma_{i}$ for each $i$ and let $\hat{\gamma}_{i}(t)$ be an estimate of $\gamma_{i}$ over all $t \geq 0$.
Assumption 3. Let $\phi_{i}(t)$ with $\phi_{i}(0) \geq 0$ be the time-varying coupling weights for all $i \in\{1,2, \ldots, N\}$ and for all $t \in \mathbb{R}_{\geq 0}$.

Assumption 4. Let $\sigma_{i}(t)$ be a uniformly continuous and bounded function [34] such that for any $t_{0} \geq 0$

$$
\lim _{t \rightarrow \infty} \int_{t_{0}}^{t} \sigma_{i}(\tau) d \tau \leq \bar{\sigma}_{i}<\infty \quad \forall i
$$

Objective of SVC is to design an appropriate control input $v_{i}$ such that $z_{i}$ approaches $z_{0} \forall i \in\{1,2, \ldots, N\}$. Motivated by [35. [36], a fully-distributed and robust SVC law is proposed below:

$$
\left\{\begin{array}{l}
v_{i}=-\left(\phi_{i}+\varphi_{i}\right) K \xi_{i}, \\
\dot{\phi}_{i}=-\kappa_{1} \sigma_{i} \phi_{i}+\kappa_{1}\left\|K \xi_{i}\right\|^{2}, \\
\dot{\hat{\gamma}}_{i}=-\kappa_{2} \sigma_{i} \hat{\gamma}+\kappa_{2}\left\|K \xi_{i}\right\|, \\
\varphi_{i}=\hat{\gamma}_{i}^{2} /\left(\hat{\gamma}_{i}\left\|K \xi_{i}\right\|+\sigma_{i}\right) \quad \forall i
\end{array}\right.
$$

where

$$
\xi_{i}=\sum_{j=1}^{N} a_{i j}\left(z_{i}-z_{j}\right)+g_{i}\left(z_{i}-z_{0}\right) \quad \forall i
$$

denotes the local voltage magnitude tracking error of the $i^{\text {th }}$ DG unit, the constants $\kappa_{1} \in \mathbb{R}_{>0}$ and $\kappa_{2} \in \mathbb{R}_{>0}$ are to be selected and $K$ is the feedback gain matrix to be designed. We denote $z=\left[z_{1}^{T}, z_{2}^{T}, \ldots, z_{N}^{T}\right]^{T}, d=\left[d_{1}, d_{2}, \ldots, d_{N}\right]^{T}$ and $\xi=\left[\xi_{1}^{T}, \xi_{2}^{T}, \ldots, \xi_{N}^{T}\right]^{T}$. Applying the SVC protocol (17), the closed-loop linearized system dynamics of $N$ DG units can be expressed in the Kronecker product form as

$$
\dot{z}=\left(I_{N} \otimes A\right) z+\left(I_{N} \otimes B\right) d-[(\Phi+\Omega) \otimes B K] \xi
$$

where $\Phi=\operatorname{diag}\left\{\phi_{1}, \ldots, \phi_{N}\right\}$ and $\Omega=\operatorname{diag}\left\{\varphi_{1}, \ldots, \varphi_{N}\right\}$. The following theorem, one of the main contributions of this paper, establishes that all $z_{i}$ in (14) synchronise to $z_{0}$ in (15) due to the action of the proposed SVC scheme, which implies that the output voltage magnitude $v_{o, m a g i}$ of all DG units will eventually attain $V_{\text {ref }}$.

Theorem 1. Consider the feedback linearized model of $N D G$ units as described in (14) assuming that the internal dynamics of each DG is asymptotically stable. Suppose further that Assumptions 14 hold. Then, the direct component of the DG output voltage $v_{\text {odi }}$ asymptotically tracks the reference voltage $V_{\text {ref }}$ for all $i \in\{1,2, \ldots, N\}$ due to application of the SVC law 17) with $K=B^{T} P$ where $P>0$ is a solution to the following algebraic Riccati (ARE) equation

$$
P A+A^{T} P-P B B^{T} P+Q=0
$$

for a given $Q>0$.

Proof. Define $\zeta_{i}=z_{i}-z_{0}$ and let the global voltage magnitude tracking error vector be $\zeta=\left[\zeta_{1}^{T}, \zeta_{2}^{T}, \ldots, \zeta_{N}^{T}\right]^{T}$. Substituting (19) into the derivative of $\zeta$, we find

$$
\dot{\zeta}=\left(I_{N} \otimes A\right) \zeta+\left(I_{N} \otimes B\right) d-\left[(\Phi+\Omega) \otimes B B^{T} P\right] \xi .
$$


Consider the following Lyapunov function candidate

$$
V_{1}=\zeta^{T}[(L+G) \otimes P] \zeta+\sum_{i=1}^{N} \frac{\tilde{\phi}_{i}^{2}}{\kappa_{1}}+\sum_{i=1}^{N} \frac{\tilde{\gamma}_{i}^{2}}{\kappa_{2}}
$$

where $\tilde{\phi}_{i}=\phi_{i}-\alpha, \tilde{\gamma}_{i}=\hat{\gamma}_{i}-\gamma_{i}$ and the constant $\alpha \in \mathbb{R}_{>0}$ to be determined later. It can be readily verified that $V_{1}$ is positive definite. We now compute the time-derivative of $V_{1}$ along the trajectories of (21)

$$
\dot{V}_{1}=2 \zeta^{T}[(L+G) \otimes P] \dot{\zeta}+2 \sum_{i=1}^{N} \frac{\tilde{\phi}_{i} \dot{\tilde{\phi}}_{i}}{\kappa_{1}}+2 \sum_{i=1}^{N} \frac{\tilde{\gamma}_{i} \dot{\tilde{\gamma}}_{i}}{\kappa_{2}} .
$$

Substituting (21) and (17) into (23), we have

$$
\begin{gathered}
\dot{V}_{1}=2 \zeta^{T}[(L+G) \otimes P A] \zeta+2 \zeta^{T}[(L+G) \otimes P B] d- \\
2 \zeta^{T}\left[(L+G)(\Phi+\Omega) \otimes P B B^{T} P\right] \xi+2 \sum_{i=1}^{N} \tilde{\phi}_{i}\left(-\phi_{i} \sigma_{i}+\right. \\
\left.\left\|B^{T} P \xi_{i}\right\|^{2}\right)+2 \sum_{i=1}^{N} \tilde{\gamma}_{i}\left(-\hat{\gamma}_{i} \sigma_{i}+\left\|B^{T} P \xi_{i}\right\|\right)
\end{gathered}
$$

Note that $\xi_{i}$ and $\zeta_{i}$ are related via the expression

$$
\xi=\left[(L+G) \otimes I_{n}\right] \zeta .
$$

It follows from the definitions of $\Phi$ and $\tilde{\phi}_{i}$ that

$$
\begin{aligned}
& -2 \zeta^{T}\left[(L+G) \Phi \otimes P B B^{T} P\right] \xi+2 \sum_{i=1}^{N} \tilde{\phi}_{i}\left\|B^{T} P \xi_{i}\right\|^{2} \\
= & -2 \sum_{i=1}^{N} \phi_{i}\left\|B^{T} P \xi_{i}\right\|^{2}+2 \sum_{i=1}^{N}\left(\phi_{i}-\alpha\right)\left\|B^{T} P \xi_{i}\right\|^{2} \\
= & -2 \alpha \zeta^{T}\left[(L+G)^{2} \otimes P B B^{T} P\right] \zeta .
\end{aligned}
$$

Assumption 2 can be applied to obtain the following relation

$$
2 \zeta^{T}[(L+G) \otimes P B] d \leq 2 \sum_{i=1}^{N} \gamma_{i}\left\|B^{T} P \xi_{i}\right\| .
$$

Now, 24) implies (28) upon substituting (26) and (27)

$$
\begin{aligned}
\dot{V}_{1} \leq & 2 \zeta^{T}[(L+G) \otimes P A] \zeta-2 \alpha \zeta^{T}\left[(L+G)^{2} \otimes P B B^{T} P\right] \zeta \\
& -2 \zeta^{T}\left[(L+G) \Omega \otimes P B B^{T} P\right] \xi+2 \sum_{i=1}^{N} \hat{\gamma}_{i}\left\|B^{T} P \xi_{i}\right\| \\
& -2 \sum_{i=1}^{N} \tilde{\phi}_{i} \phi_{i} \sigma_{i}-2 \sum_{i=1}^{N} \tilde{\gamma}_{i} \hat{\gamma}_{i} \sigma_{i} .
\end{aligned}
$$

Furthermore, from Assumptions 3 and 4 , we have

$$
\begin{aligned}
& -2 \zeta^{T}\left[(L+G) \Omega \otimes P B B^{T} P\right] \xi+2 \sum_{i=1}^{N} \hat{\gamma}_{i}\left\|B^{T} P \xi_{i}\right\| \\
= & 2 \sum_{i=1}^{N} \frac{\hat{\gamma}_{i}\left\|B^{T} P \xi_{i}\right\| \sigma_{i}}{\hat{\gamma}_{i}\left\|B^{T} P \xi_{i}\right\|+\sigma_{i}} \leq 2 \sum_{i=1}^{N} \sigma_{i} .
\end{aligned}
$$

Note that $-\tilde{\phi}_{i} \phi_{i}=-\tilde{\phi}_{i}^{2}-\tilde{\phi}_{i} \alpha \leq(1 / 4) \alpha^{2}$ and $-\tilde{\gamma}_{i} \hat{\gamma}_{i} \leq(1 / 4) \gamma_{i}^{2}$. Now, using (29), inequality 28) implies

$$
\begin{aligned}
\dot{V}_{1} \leq & 2 \zeta^{T}[(L+G) \otimes P A] \zeta-2 \alpha \zeta^{T}\left[(L+G)^{2} \otimes P B B^{T} P\right] \zeta \\
& +\sum_{i=1}^{N} k_{i} \sigma_{i}
\end{aligned}
$$

where $k_{i}=4+\left(\alpha^{2} / 2\right)+\left(\gamma_{i}^{2} / 2\right)>0$ is constant for all $i$. Now, since $L+G>0$ via Assumption 1, there exists a unitary diagonalizing matrix $T$ such that $J=\operatorname{diag}\left\{\lambda_{1}, \ldots, \lambda_{N}\right\}=$ $T^{-1}(L+G) T$. Let $\vartheta=\left(T^{-1} \otimes I_{N}\right) \zeta=\left[\vartheta_{1}^{T}, \vartheta_{2}^{T}, \ldots, \vartheta_{N}^{T}\right]^{T}$ and subsequently (30) is expressed in terms of $\vartheta$ as

$$
\dot{V}_{1} \leq \vartheta^{T}\left[J \otimes\left(P A+A^{T} P\right)-2 \alpha J^{2} \otimes P B B^{T} P\right] \vartheta+\sum_{i=1}^{N} k_{i} \sigma_{i}
$$

which is further simplified to

$$
\dot{V}_{1} \leq \sum_{i=1}^{N} \lambda_{i} \vartheta^{T}\left(P A+A^{T} P-2 \alpha \lambda_{i} P B B^{T} P\right) \vartheta+\sum_{i=1}^{N} k_{i} \sigma_{i} .
$$

By choosing a sufficiently large $\alpha$ such that $\alpha \geq \frac{1}{2 \lambda_{\min }(L+G)}$, it follows from the ARE given in 20, that

$$
\dot{V}_{1} \leq-\sum_{i=1}^{N} \lambda_{i} \vartheta^{T} Q \vartheta+\sum_{i=1}^{N} k_{i} \sigma_{i} \leq-\underline{\lambda}\|\vartheta\|^{2}+\sum_{i=1}^{N} k_{i} \sigma_{i}
$$

where $\underline{\lambda}=\lambda_{\min }(L+G) \lambda_{\min }(Q)$. Now, integrating both sides of (32) with respect to $t$ from 0 to $T \geq 0$, we have

$$
\begin{aligned}
\left.V_{1}\right|_{t=T} \leq\left. V_{1}\right|_{t=0}-\int_{0}^{T} \underline{\lambda}\|\vartheta(t)\|^{2} \mathrm{~d} t+\int_{0}^{T} \sum_{i=1}^{N} k_{i} \sigma_{i}(t) \mathrm{d} t \\
\left.\Rightarrow V_{1}\right|_{t=T} \leq\left. V_{1}\right|_{t=0}+\sum_{i=1}^{N} k_{i} \bar{\sigma}_{i} \quad[\text { since } \underline{\lambda}>0]
\end{aligned}
$$

where $\left.V_{1}\right|_{t=T}$ denotes $V\left(\vartheta(T), \tilde{\phi}_{i}(T), \tilde{\gamma}_{i}(T)\right)$ for any $T \geq 0$. Since $\left.V_{1}\right|_{t=0}$ is constant for a given set of initial conditions and $\bar{\sigma}_{i}$ are finite and time independent, the function $V_{1}$ remains uniformly bounded, that is for all $t \geq 0$, which implies that $\vartheta_{i}(t), \tilde{\phi}_{i}(t)$ and $\tilde{\gamma}_{i}(t)$ are all uniformly bounded for each $i$. Moreover, since $\left.V_{1}\right|_{t=T} \geq 0$ for all $T \geq 0$, the inequality 33a implies

$$
\int_{0}^{T} \underline{\lambda}\|\vartheta(t)\|^{2} \mathrm{~d} t \leq\left. V_{1}\right|_{t=0}+\sum_{i=1}^{N} k_{i} \bar{\sigma}_{i} \quad \forall T \geq 0
$$

which implies $\vartheta(t) \in \mathbb{L}_{2} \bigcap \mathbb{L}_{\infty}$. Since $\vartheta(t)=\left(T^{-1} \otimes I_{N}\right) \zeta(t)$, $\zeta(t) \in \mathbb{L}_{2} \cap \mathbb{L}_{\infty}$ which in turn implies $\xi(t)=\left[(L+G) \otimes I_{N}\right] \zeta(t) \in$ $\mathbb{L}_{2} \cap \mathbb{L}_{\infty}$. Therefore, $\dot{\zeta}(t) \in \mathbb{L}_{\infty}$ from (21) since $\zeta(t), d(t)$ and $\xi(t)$ are all $\mathbb{L}_{\infty}$ bounded signals $\forall t \geq 0$. Now invoking Lemma 3. it can be ensured that $\lim _{t \rightarrow \infty} \zeta(t)=0$, which means

$$
\lim _{t \rightarrow \infty}\left[z_{i}(t)-z_{0}(t)\right]=0 \quad \forall i .
$$

Hence, $v_{\text {odi }}$ asymptotically reaches $V_{\text {ref }}$ for each $i \in$ $\{1,2, \ldots, N\}$ under the assumption that the internal dynamics of all DG units are asymptotically stable.

The block diagram of the proposed fully-distributed and robust SVC scheme is depicted in Fig. 2 


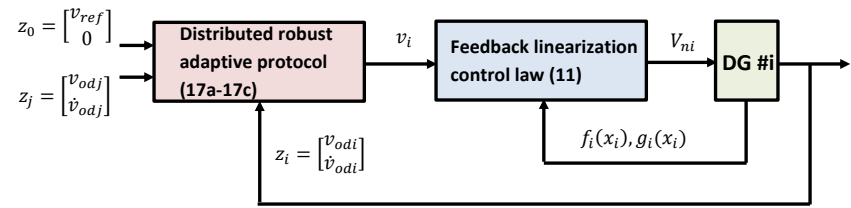

Figure 2: Functional block diagram of the SVC scheme applied to the $i^{\text {th }}$ DG unit.

\subsection{Distributed and robust secondary frequency control}

Similar to SVC, in this subsection, our aim is to develop a robust and distributed secondary frequency control (SFC) technique that will guarantee frequency synchronisation of all DG units to the nominal grid frequency $\left(\omega_{\text {ref }}\right)$. Here, the output is denoted by $y_{2 i}=\omega_{i}$ and the control input is $u_{2 i}=\omega_{n i}$. Applying again the input-output feedback linearization tool on (7a)-(7c) considering $y_{2 i}=\omega_{i}$ as the output, we obtain a first-order dynamics

$$
\dot{\omega}_{i}=\dot{\omega}_{n i}-m_{P i} \dot{P}_{i} \quad \forall i .
$$

The auxiliary control input $v_{2 i}$ is defined as

$$
v_{2 i}=\dot{\omega}_{n i}-m_{P i} \dot{P}_{i} \quad \forall i
$$

such that 36 gets transformed into a single integrator dynamics

$$
\dot{\omega}_{i}=v_{2 i} \quad \forall i \in\{1,2, \ldots, N\} .
$$

The relative frequency tracking error for each of the DG units is defined as

$$
\xi_{\omega i}=\sum_{j=1}^{N} a_{i j}\left(\omega_{i}-\omega_{j}\right)+g_{i}\left(\omega_{i}-\omega_{r e f}\right) .
$$

Being inspired from [37], the following adaptive control law is designed to achieve the secondary frequency control (SFC)

$$
\left\{\begin{aligned}
v_{2 i} & =\left(c_{i}+\rho_{i}\right) K_{\omega} \xi_{\omega i} \quad \forall i \in\{1,2, \ldots, N\}, \\
\dot{c}_{i} & =Q_{\omega} \xi_{\omega i}^{2},
\end{aligned}\right.
$$

where $c_{i}(t)$ with $c_{i}(0)>0$ represent the time-varying coupling weights; $K_{\omega}$ and $Q_{\omega}$ are positive constants to be selected; and $\rho_{i}$ is a set of smooth functions to be determined. Let us denote $\omega=\left[\omega_{1}, \ldots, \omega_{N}\right]^{T}$ and the global frequency tracking error $\xi_{\omega}=$ $\left[\xi_{\omega 1}, \ldots, \xi_{\omega N}\right]^{T}$. From [39], $\xi_{\omega}$ can be expressed in a compact form as $\left(\mathbf{1}_{N}\right.$ denotes a column vector of all 1$)$

$$
\xi_{\omega}=(L+G)\left(\omega-\mathbf{1}_{N} \omega_{r e f}\right) .
$$

Substituting (36) and (40) into the derivative of (41), the closedloop frequency tracking error dynamics is obtained as

$$
\left\{\begin{array}{l}
\dot{\xi}_{\omega}=K_{\omega}(L+G)(C+\rho) \xi_{\omega} \\
\dot{c}_{i}=Q_{\omega} \xi_{\omega i}^{2}
\end{array}\right.
$$

where $C=\operatorname{diag}\left\{c_{1}, \ldots, c_{N}\right\}$ and $\rho=\operatorname{diag}\left\{\rho_{1}, \ldots, \rho_{N}\right\}$.
Theorem 2. Consider the feedback linearized model (38) assuming that the internal dynamics of each DG is asymptotically stable. Then, the frequency $\omega_{i}$ of each DG unit asymptotically reaches the nominal frequency $\omega_{\text {ref }}$ subjected to the SFC law (40) with $K_{\omega}=-\sqrt{\frac{Q_{\omega}}{R_{\omega}}}$ and $\rho_{i}=\sqrt{Q_{\omega} R_{\omega}} \xi_{\omega i}^{2}$ for given $R_{\omega}>0$ and $Q_{\omega}>0$.

Proof. As $(L+G)$ is a non-singular $M$-matrix via Assumption 1. there exists $\Xi=\operatorname{diag}\left\{\psi_{i}\right\}$ with $\psi_{i}>0$ for all $i \in\{1,2, \ldots, N\}$ such that $\Xi(L+G)+(L+G)^{T} \Xi>0$ according to Lemma 2. In this proof, the SFC problem has been posed as an asymptotic stability problem of the closed-loop system (42). We start with an appropriate Lyapunov function candidate

$$
V_{2}=\sum_{i=1}^{N} \frac{1}{2} \psi_{i}\left(2 c_{i}+\rho_{i}\right) \rho_{i}+\frac{1}{2} \sum_{i=1}^{N} \psi_{i}\left(c_{i}-\beta\right)^{2}
$$

where $\beta>0$ is a constant to be determined later and $\dot{c}_{i}(t) \geq$ 0 with $c_{i}(0)>0$ implies $c_{i}(t)>0$ for all $t>0$. It can be verified that $V_{2}>0$. Now, the time derivative of $V_{2}$ along the trajectories of (42) is computed as

$$
\begin{aligned}
\dot{V}_{2} & =\sum_{i=1}^{N}\left[\psi_{i}\left(c_{i}+\rho_{i}\right) \dot{\rho}_{i}+\psi_{i} \rho_{i} \dot{c}_{i}\right]+\sum_{i=1}^{N} \psi_{i}\left(c_{i}-\beta\right) \dot{c}_{i} \\
& =\sum_{i=1}^{N} 2 \psi_{i}\left(c_{i}+\rho_{i}\right) \xi_{\omega i} \sqrt{Q_{\omega} R_{\omega}} \dot{\xi}_{\omega i}+\sum_{i=1}^{N} \psi_{i}\left(\rho_{i}+c_{i}-\beta\right) \dot{c}_{i} .
\end{aligned}
$$

Note that

$$
\begin{aligned}
& \sum_{i=1}^{N} \psi_{i}\left(\rho_{i}+c_{i}-\beta\right) \dot{c}_{i}=\xi_{\omega}^{T}\left[Q_{\omega}(C+\rho-\beta I) \Xi\right] \xi_{\omega} \\
& \text { and } \sum_{i=1}^{N} 2 \psi_{i}\left(c_{i}+\rho_{i}\right) \xi_{\omega i} \sqrt{Q_{\omega} R_{\omega}} \dot{\xi}_{\omega i} \\
&=2 \xi_{\omega}\left[\sqrt{Q_{\omega} R_{\omega}}(C+\rho) \Xi\right] \dot{\xi}_{\omega} \\
&=-\xi_{\omega}^{T}\left[Q_{\omega}(C+\rho)\left(\Xi(L+G)+(L+G)^{T} \Xi\right)(C+\rho)\right] \xi_{\omega} \\
& \leq-\xi_{\omega}^{T}\left[\lambda_{1, \min } Q_{\omega}(C+\rho)^{2}\right] \xi_{\omega}
\end{aligned}
$$

where $\lambda_{1, \min }$ indicates the smallest positive eigenvalue of $\left[\Xi(L+G)+(L+G)^{T} \Xi\right]$. Exploiting conditions (46) and 45], Lyapunov inequality (44) implies

$$
\dot{V}_{2} \leq \xi_{\omega}^{T}\left[Q_{\omega}(C+\rho) \Xi-Q_{\omega}\left(\lambda_{1, \min }(C+\rho)^{2}+\beta \Xi\right)\right] \xi_{\omega} .
$$

The following implication

$$
\begin{aligned}
& -\xi_{\omega}^{T}\left[Q_{\omega}\left(\lambda_{1, \min }(C+\rho)^{2}+\beta \Xi\right)\right] \xi_{\omega} \\
\leq & -2 \xi_{\omega}^{T}\left[Q_{\omega} \sqrt{\lambda_{1, \min } \beta \Xi}(C+\rho)\right] \xi_{\omega}
\end{aligned}
$$

holds due to the property $X^{2}+Y^{2} \geq 2 X Y$ when $X=X^{T} \geq 0$ and $Y=Y^{T} \geq 0$. Selecting $\beta \geq \frac{\max _{i \in\{1, \ldots, N\}} \varphi_{i}}{\lambda_{1, \min }}$ in (48) and applying the result into (47), we obtain

$$
\dot{V}_{2} \leq-\xi_{\omega}^{T}\left[Q_{\omega}(C+\rho) \Xi\right] \xi_{\omega} .
$$




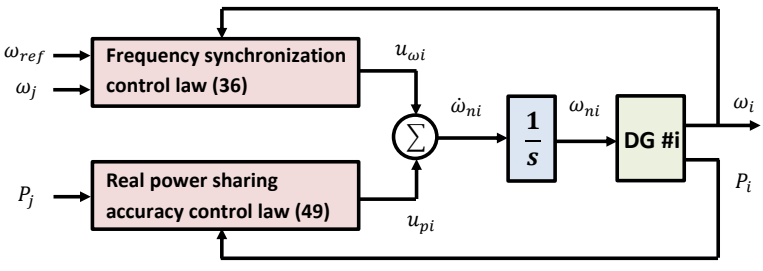

Figure 3: Functional block diagram of the SFC scheme applied to the $i^{\text {th }}$ DG unit.

Applying the change of variable

$$
\zeta_{\omega}=\sqrt{(C+\rho) \Xi} \xi_{\omega}
$$

into [49], the expression for $\dot{V}_{2}$ becomes

$$
\dot{V}_{2} \leq-Q_{\omega} \zeta_{\omega}^{T} \zeta_{\omega}
$$

which implies $\dot{V}_{2} \leq 0$ and $\dot{V}_{2}=0$ only when $\zeta_{\omega}=0$. This implies asymptotic stability of (42) invoking LaSalle's invariance principle [34]. This ensures $\lim _{t \rightarrow \infty} \zeta_{\omega}(t)=0$ and in turn,

$$
\lim _{t \rightarrow \infty} \xi_{\omega}(t)=0
$$

since $\zeta_{\omega}$ and $\xi_{\omega}$ are related via non-singular transformation (50). Hence, $\omega_{i}$ approaches $\omega_{\text {ref }}$ for all $i \in\{1,2, \ldots, N\}$ as $t \rightarrow \infty$. This completes the proof.

The block diagram of the proposed fully-distributed and robust SFC scheme is shown in Fig. 3 .

Remark 2. The scalar control parameters $Q_{\omega}>0$ and $R_{\omega}>$ 0 used in the SFC protocol (40) have a direct impact on the transient response of the $D G$ unites. In order to get a faster response of $\omega_{i}$, a larger $Q_{\omega}$ and a smaller $R_{\omega}$ can be chosen. But that may increase the control input demand and in practical designs, control input may have to be restricted due to physical limitations of the actuators. To maintain a trade-off between the speed of response and the required control effort, difference between $Q_{\omega}$ and $R_{\omega}$ should not be too high and the design also depends on other performance constraints.

\subsection{Real power sharing among the DG units}

Note that the real power sharing between two DG units is taken care by primary control according to the relationship

$$
P_{1} m_{p 1}=P_{2} m_{p 2}=\cdots=P_{N} m_{p N} .
$$

In order to ensure real power sharing among the DG units satisfying (53) along with secondary voltage and frequency control, we introduce an additional control input component

$$
u_{p i}=m_{P i} \dot{P}_{i} \quad \forall i .
$$

The consensus error of the active power transferred by the $i^{\text {th }}$ DG is defined by

$$
\xi_{p i}=\sum_{j=1}^{N} a_{i j}\left(m_{P i} P_{i}-m_{P j} P_{j}\right) \quad \forall i .
$$

Similar to the previous section, an adaptive control protocol is selected below to facilitate real power sharing:

$$
\left\{\begin{array}{l}
u_{p i}=\left(c_{p i}+\rho_{p i}\right) K_{p} \xi_{p i}, \\
\dot{c}_{p i}=Q_{p} \xi_{p i}^{2}, \quad \forall i
\end{array}\right.
$$

where $K_{p}=-\sqrt{\frac{Q_{p}}{R_{p}}}$ and $\rho_{p i}=\sqrt{Q_{p} R_{p}} \xi_{p i}^{2}$ with $Q_{p} \in \mathbb{R}_{>0}$ and $R_{p} \in \mathbb{R}_{>0}$ being the control parameters. Following the proof of Theorem 2, it can be readily established that active power outputs $\left(P_{i}\right)$ of the DG units will be governed by the ratio $\frac{1}{m_{p 1}}$ : $\frac{1}{m_{p 2}}: \cdots: \frac{1}{m_{p N}}$ by the proposed distributed control law 56 . Substituting (54) into (36), the combined control input $\omega_{n i}$ is obtained as

$$
\omega_{n i}=\int\left(u_{\omega i}+u_{p i}\right) \mathrm{d} t \quad \forall i .
$$

$\omega_{n i}$ is the generated reference input to the primary control block (see Fig. 1), which is responsible for frequency synchronisation of all DG units in a microgrid as well as the active power sharing among them at a particular ratio during the islanded mode of operation.

Remark 3. The fundamental difference between our approach and that of the literature [26, 21, 24, 25] is that we have used a two-layer control scheme in which the inner layer first applies a feedback linearisation technique to transform the nonlinear and coupled dynamics of the microgrid into Brunovsky normal form and then the outer layer implements the SVFC protocols to synchronise the voltage and frequency of each DG to the nominal values exploiting consensus principle. On the contrary, in [21 24], control laws are designed by utilising the distributed averaging (DAPI) technique and manipulate directly the droop characteristic equations $\omega_{i}=\omega^{*}-m_{i} P_{i}$ and $v_{i}=v_{i}^{*}-n_{i} Q_{i}$. In [22], a small-signal approximated model is first obtained and then a optimal static output feedback control law is designed. It is mentioned in [21] that complete closed-loop stability analysis in the SVC case is extremely challenging and hence, a smallsignal stability analysis has been resorted in [21]. Whereas the present two-layer control scheme involving feedback linearisation facilitates a complete stability analysis framework via Lyapunov approach for both secondary voltage control and frequency control along with real power sharing.

\section{Case study on a prototype microgrid system}

To show the effectiveness of the proposed fully-distributed and robust SVFC scheme, a prototype microgrid system with five DG units interconnected via IEEE 14-bus configuration [38] is considered in Fig. 4. Initial loads of all five DG units are given by $1.5 \mathrm{~kW}+1.2 \mathrm{kVAr}, 2 \mathrm{~kW}+0.6 \mathrm{kVAr}, 0.8 \mathrm{~kW}+0.8 \mathrm{kVAr}$, $1.6 \mathrm{~kW}+1.3 \mathrm{kVAr}$ and $1.1 \mathrm{~kW}+1.5 \mathrm{kVAr}$. The real power sharing ratio is specified as $1: 2: 3: 4: 5$. The simulation is carried out in Matlab-Simulink environment. The SVC parameters are chosen as: $\sigma_{i}=2 e^{-2 t}, \phi_{i}(0)=0, \hat{\gamma}_{i}(0)=2 \forall i \in\{1, \ldots, 5\}$, $\kappa_{1}=\kappa_{2}=1$ and $Q=\left[\begin{array}{cc}1000 & 0 \\ 0 & 10\end{array}\right]$. Note, $\sigma_{i}$ is uniformly continuous and bounded. Solving ARE 20, We find a $P>0$ which yields $K=B^{T} P=\left[\begin{array}{ll}31.6228 & 8.5584\end{array}\right]$. The SFC parameters 


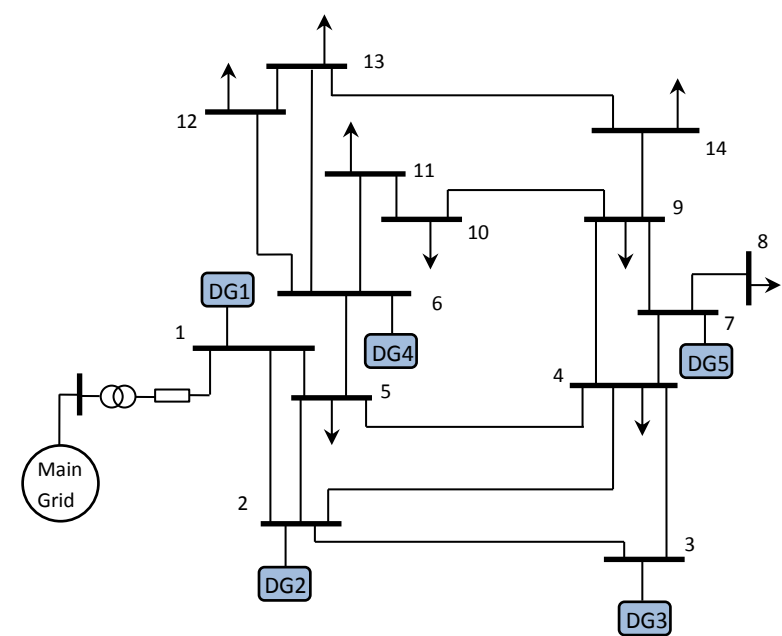

Figure 4: A prototype IEEE 14-bus microgrid system containing five DG units.

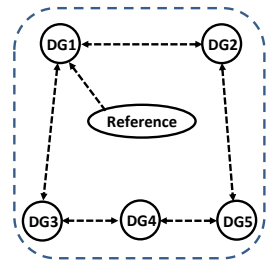

(a)

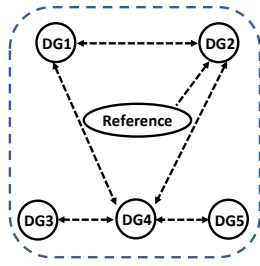

(b)

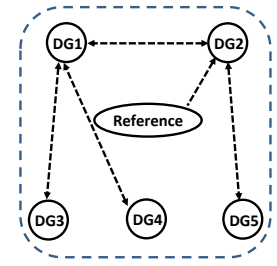

(c)
Figure 5: Time-varying communication graphs of the DG units.

are considered to be $Q_{\omega}=300, R_{\omega}=0.01, Q_{p}=100$ and $R_{p}=1$. The nominal grid voltage and frequency are set as $V_{\text {ref }}=310 \mathrm{~V}$ and $\omega_{\text {ref }}=100 \pi \mathrm{rad} / \mathrm{s}$.

\subsection{Voltage and frequency restoration with real power sharing}

In the first phase $(0 \leq t<10$ s) of simulation, secondary control has not been activated. It is apparent from Fig. 6 that primary control action has not been able to achieve perfect synchronisation of $v_{o i}$ and $\omega_{i}$ to $V_{\text {ref }}$ and $\omega_{\text {ref }}$. At $t=10 \mathrm{~s}$, both SVC and SFC schemes are activated together along with 56 to ensure active power sharing. The simulation results suggest that the proposed SVFC scheme is able to prevent any deviations in the voltage and frequency levels of individual DG units, that is, $v_{o i}$ reaches $310 \mathrm{~V}$ and $\omega_{i}$ reaches $100 \pi \mathrm{rad} / \mathrm{sec}($ i.e., $50 \mathrm{~Hz}$ ) for all $i$ under the influence of the developed SVFC scheme. Note that the responses are quite smooth with minimum transients. This happens primarily due to the effect of the feedback linearising controller since it compensates for most of the nonlinear terms present in the plant dynamics and makes the final response achieved by the secondary controller smoother.

Remark 4. In the proposed methodology, all three distributed control protocols SVC, SFC and (56) to facilitate real power sharing among the DG units work simultaneously and independent of each other to achieve voltage and frequency synchronisation for each DGs along with maintaining the real power sharing among them at a pre-specified ratio. Simulation results
(Fig. 7) show that the proposed SVFC control scheme has met all these objectives with satisfactory transient performance under the nominal operating condition of the islanded microgrid as well as in the event of topology changes and during plugand-play operation.

\subsection{Robustness of the proposed SVFC scheme to frequent com- munication topology changes, load variations and plug- and-play operation}

In order to test the robust performance of the proposed SVFC scheme, the communication graph is switched in the interval of 40s following a sequence (a) $\rightarrow$ (b) $\rightarrow$ (c) $\rightarrow$ (a) as shown in Figures 5(a) 5(c) staring from $t=40 \mathrm{~s}$. Fig. 6 shows that despite frequent switching in the communication network, the output voltage and frequency of each DG unit are tracking the reference values without any steady-state error. Apart from voltage and frequency synchronisation, active power $\left(P_{i}\right)$ produced by the DG units are found to be in the desired ratio $P_{1}: P_{2}: P_{3}: P_{4}: P_{5}=1 / m_{p 1}: 1 / m_{p 2}: 1 / m_{p 3}: 1 / m_{p 4}:$ $1 / m_{p 5}=1: 2: 3: 4: 5$ for all $t>10$ s and irrespective of any load fluctuations, due to the influence of the SVFC scheme. Fig. 6 also shows that the DGs generate reactive power $\left(Q_{i}\right)$ in the same ratio as that of the active power $\left(P_{i}\right)$ generation. Furthermore, in order to evaluate robust performance of the proposed scheme, an additional load $L_{c}=1 \mathrm{~kW}+0.8 \mathrm{kVAr}$ is assigned to DG3 at $t=40 \mathrm{~s}$. It is observed from Fig. 6 that, due to this additional loading, not only DG3 but the remaining DG units also have increased their active power generation to maintain the specified real power sharing ratio. The reactive power generation for all DG units has also increased. Note that the output voltages and frequencies of the DGs are still synchronised (after the transients die down) to the nominal level due to the secondary control action. 'Plug-and-play' capability of the proposed SVFC scheme is examined by disconnecting DG5 from the network at $t=80 \mathrm{~s}$ and reconnecting it at $t=120 \mathrm{~s}$. The simulation results (Fig. 6) indicate that active power generated by DG5 reduces to zero (green plot) immediately after taking out DG5 from the network and remains zero until DG5 is plugged into the network at $t \geq 120 \mathrm{~s}$. During this period, the remaining DG units generate excess active power in the ratio $1: 2: 3: 4$ to compensate for DG5. This phenomena is clearly reflected in Fig. 6. Most importantly, there is no collapse in the voltage and frequency profile of any of the DG units due to this unprecedented disturbance happened because of disconnecting a live DG unit from the network. Note that when some DG units suffer from communication link failure, the remaining units connected to the network can still achieve consensus due to application of the proposed fully-distributed SVFC protocol and hence, guarantees reliable and safe operation of the whole microgrid in islanded mode.

Remark 5. Topology switching and plug-and-play operation give rise to a modified interaction topology among the DGs and hence a new Laplacian matrix. But the proposed distributed SVFC control protocols (Theorems 1 and 2) do not explicitly depend on the graph Laplacian matrix $(L+G)$ and therefore, the scheme continues to work even when there is a change in the 

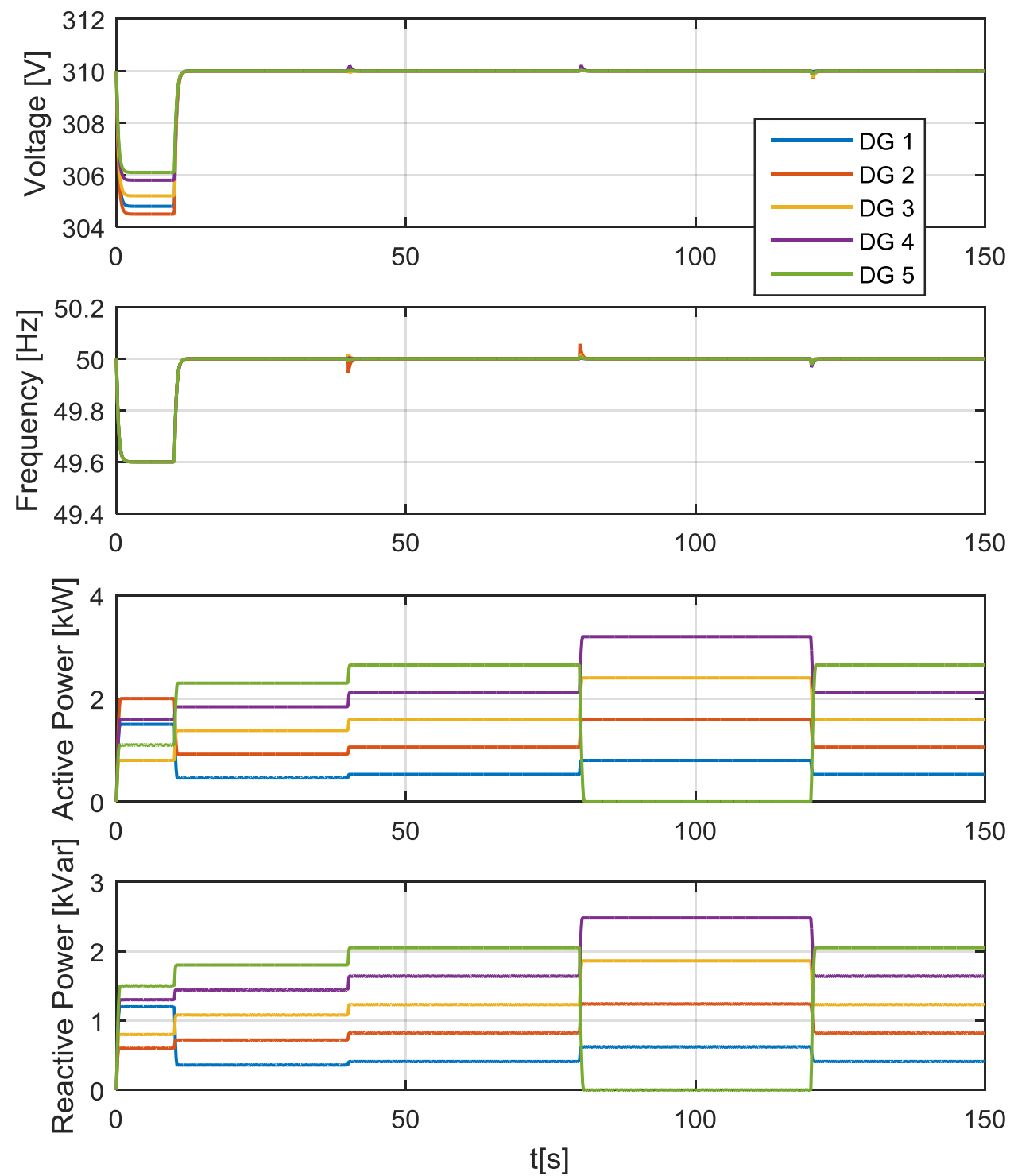

Figure 6: Simulation responses of the prototype microgrid system employing the SVFC scheme with real power sharing: (a) Output voltage magnitudes $v_{o, m a g i}$, (b) frequencies $\omega_{i}$, (c) active power delivered $P_{i}$ and (d) reactive power $Q_{i}$ delivered of all five DG units operating in islanded mode. At $t=0^{+}$, the microgrid is disconnected from the main grid and for $0<t<10 \mathrm{~s}$, the primary controller works alone. At $t=10 \mathrm{~s}$, the SVFC scheme is triggered and for $10 \leq t \leq 40$ nominal operating condition has been maintained. From $t=40 \mathrm{~s}$ onwards, the communication topology has been switched in the interval of $40 \mathrm{~s}$. Furthermore, at $t=40 \mathrm{~s}$, an additional load is assigned on DG3 and at $t=80 \mathrm{~s}$, DG5 has been disconnected from the network and reconnected at $t=120 \mathrm{~s}$ to test the plug-and-play capacity.

configuration of the DG network. In Theorem 1 the distributed control law is constructed based on the feedback linearized double-integrator dynamics given in the Brunovsky canonical form with the stabilizable pair $(A, B)$ as derived in (14). The matrices $A$ and $B$ are fixed irrespective of any changes in the topology of the DGs and accordingly, the optimal state feedback gain $K=B^{T} P$, where $P>0$ is an unique solution to the $A R E$ 20] for a given $Q>0$, also remains fixed.

\subsection{Impact of communication delays}

Communication delay is inevitable in practical applications involving communication network and sometimes causes major issues in the controller performance which may even lead to instability. In this part of the case study, we are interested to mea- sure the impact of communication delay on the performance of the microgrid achieved by the proposed SVFC scheme. Fig. 7 reveals that the closed-loop system responses $\left(v_{o i}\right.$ and $\left.P_{i}\right)$ become oscillatory as the delay increases beyond a certain extent. However, the proposed SVFC control scheme is able to preserve stability of the overall microgrid system, synchronises the output voltage and frequency of all DG units to the nominal level and also facilitates active and reactive power sharing among the DG units at the pre-specified ratio.

Remark 6. The effect of communication delay can be theoretically analysed in a similar manner as done in [22] exploiting the property that, for average consensus, the time delay should be in the range $\left(0, \tau^{*}\right)$ where $\tau^{*} \propto \frac{1}{\lambda_{\max }[L+G]}$ according to [10. 12]. It indicates that to improve the delay margin of 

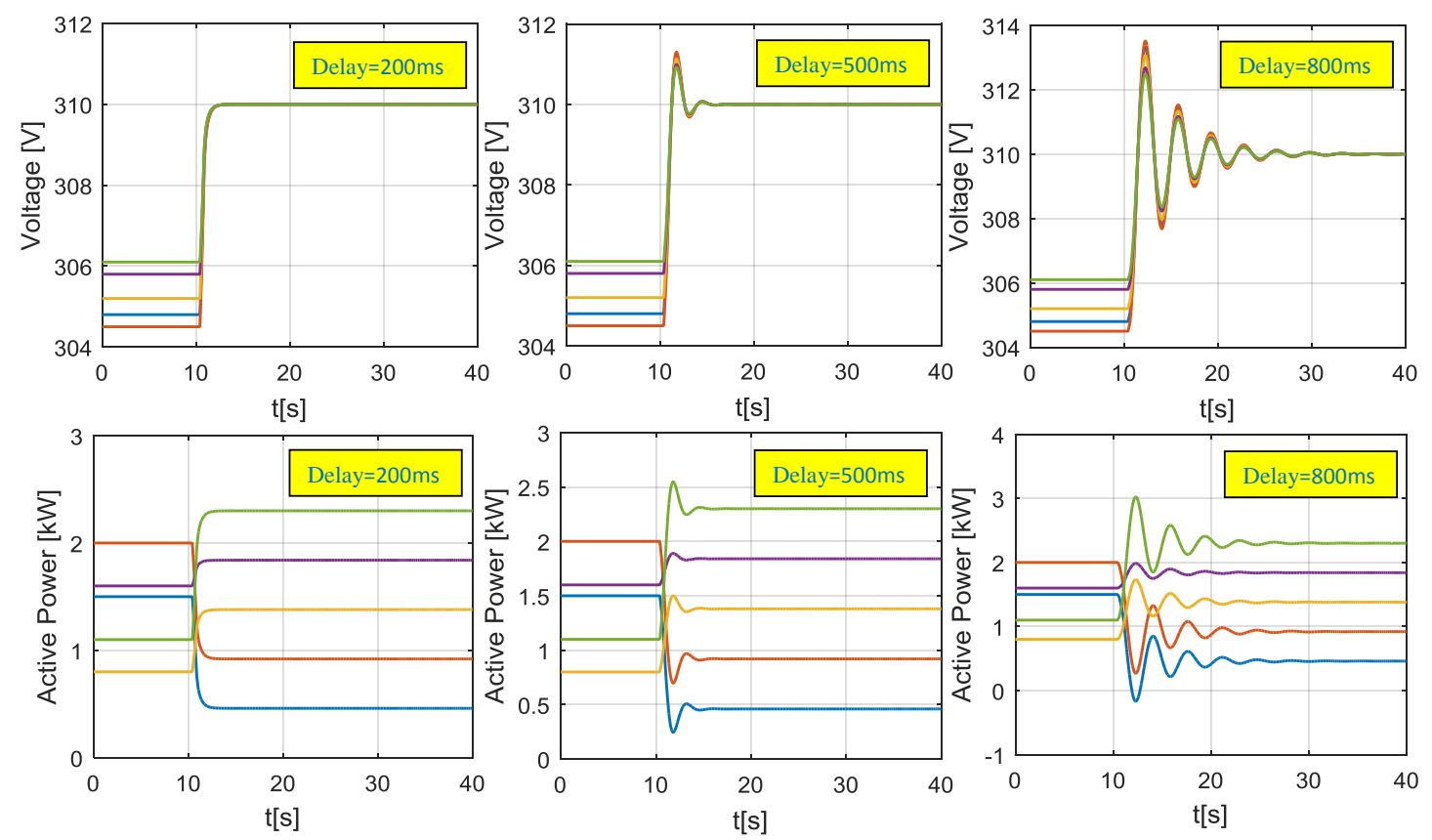

Figure 7: Output voltages $\left(v_{o i}\right)$ and active power generated $\left(P_{i}\right)$ of all five DG units subjected to $200 \mathrm{~ms}, 500 \mathrm{~ms}$ and $800 \mathrm{~ms}$ communication delay.

a network involving single or double integrator MAS, the link weightage $a_{i j}$ should be designed such that $\lambda_{\max }[L+G]$ is not too high. This analysis holds in the present scenario for the $S F C$ control protocol proposed in (40) since $K_{w}=-\frac{Q_{w}}{R_{w}}$ is constant for all $t \geq 0$ and the adaptive gains $c_{i}, \rho_{i}$ reach steady and finite values within finite time. Similar arguments hold for the SVC control law given in (17). However, detailed theoretical analysis of delay-dependent network stability is beyond the scope of this paper.

\subsection{Comparison between the proposed SVFC scheme and the scheme used in [17]}

To this end, we compare the performance achieved by the proposed distributed and robust SVFC scheme with that obtained by the controller reported in [17]. The cooperative control law of [17] is applied on the present prototype microgrid system (Fig. 4) and the parameters in the present case study are kept the same as that of [17]. Figures $8(\mathrm{a}) 8(\mathrm{~d})$ show that the performance achieved by the SVFC scheme follows closely that of [17] and it is also noticed that steady-state performance of the SVFC scheme is better (less oscillatory) than that of [17]. Note that the consensus-based scheme introduced in [17] requires to explicitly compute the non-zero minimum eigenvalue of the graph Laplacian and hence, needs to access the information of all DG units. On the contrary, the proposed control framework in this paper is fully-distributed and does not need to capture the global information about all units which enables the scheme to efficiently handle the situations when some nodes (i.e. DG units) are added to or removed from a network.

\section{Conclusion}

In this paper, multi-agent cooperative control strategies have been applied to design a fully-distributed, robust and adaptive secondary voltage and frequency control (SVFC) scheme for islanded microgrids. In contrast to the existing results on secondary controller design, the proposed control scheme is developed based on a complete nonlinear dynamic model of the inverter-based DG units (which may not be all identical) and takes into account the effects of model uncertainties, plant parameter variations and unmodelled dynamics. It is observed from the case study that the proposed SVFC scheme facilitates satisfactory transient and steady-state performance in presence of load fluctuation, plug-and-play operation, graph topology switching due to network failure and communication delays. Furthermore, the proposed secondary control framework facilitates active and reactive power sharing among the DG units apart from counteracting any deviation in the terminal voltage and frequency of each DG unit from the nominal (or reference) level. In future scope, the proposed secondary control scheme can be extended to address the network related issues, such as, data loss, packet jamming, link failure, cyber attack, etc., using Cyber Physical Systems framework [39].

\section{Acknowledgement}

The authors would like to convey sincere thanks to the Associate Editor and the anonymous Reviewers for giving constructive comments and suggestions that have enriched the theoretical contributions of the paper and also made the clarity better. The second author would also like to acknowledge Dr. Arnab Dey, Department of Electrical Engineering, IIT Kharagpur, In- 


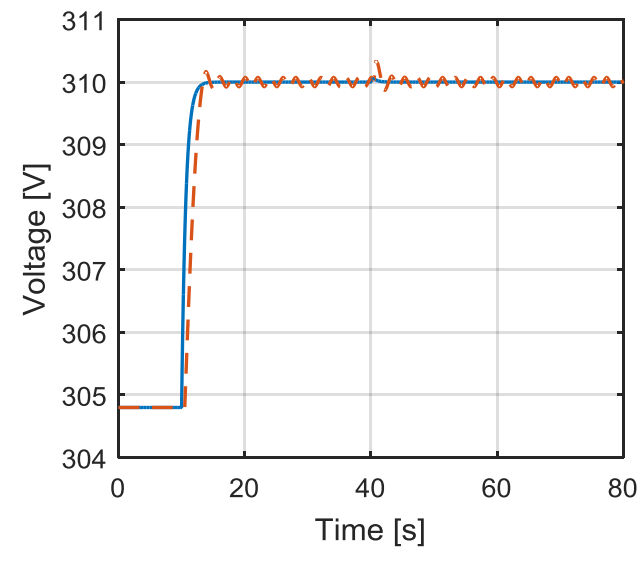

(a)

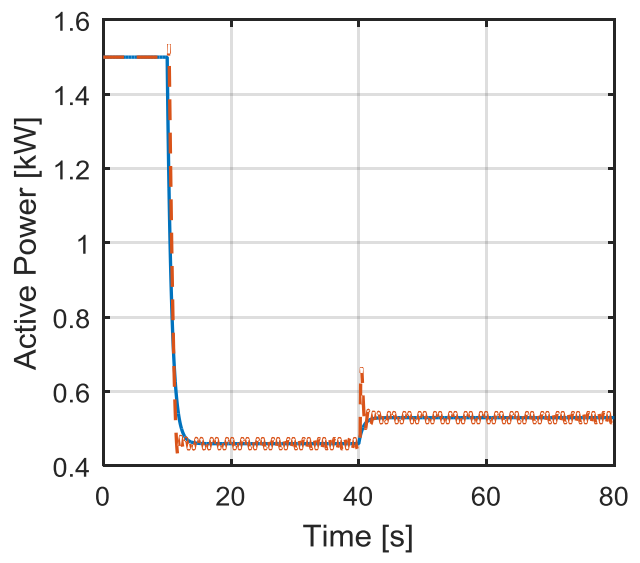

(c)

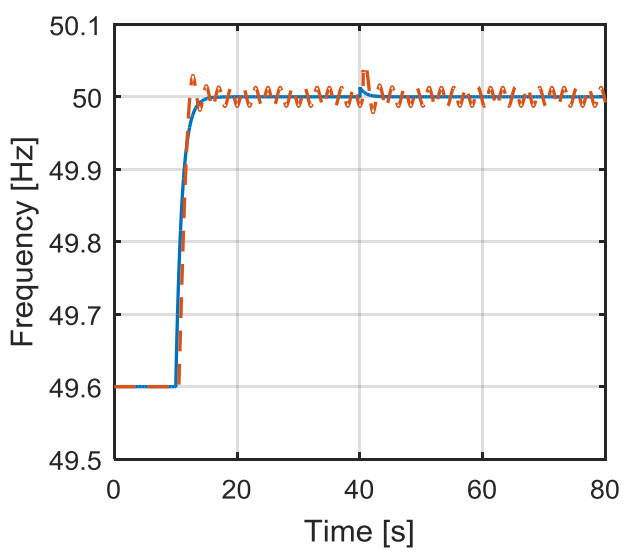

(b)

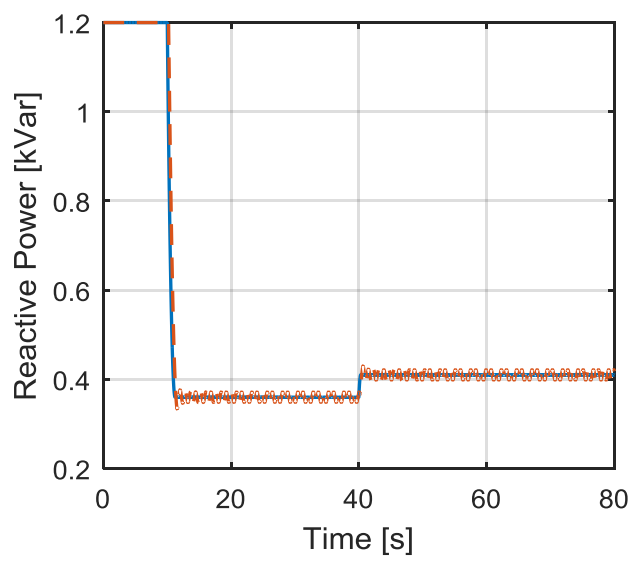

(d)

Figure 8: A comparative study of the responses: (a) voltage $v_{o 1}$ (b) frequency $\omega_{1}$, (c) active power $P_{1}$, and (d) reactive power $Q_{1}$, of DG1 achieved by the proposed SVFC scheme and the controller used in [17] (Legend: 'blue solid line': proposed controller and 'red dashed line': controller of [17]).

dia, for many useful technical discussions on Lyapunov stability analysis.

\section{References}

[1] A. Bidram, F. L. Lewis, A. Davoudi, Distributed control systems for small-scale power networks: Using multiagent cooperative control theory, IEEE Control Systems 34 (6) (2014) 56-77.

[2] F. Guo, C. Wen, J. Mao, Y.-D. Song, Distributed secondary voltage and frequency restoration control of droop-controlled inverter-based microgrids, IEEE Transactions on Industrial Electronics 62 (7) (2015) 43554364.

[3] A. Bidram, A. Davoudi, F. L. Lewis, S. S. Ge, Distributed adaptive voltage control of inverter-based microgrids, IEEE Transactions on Energy Conversion 29 (4) (2014) 862-872.

[4] N. Pogaku, M. Prodanovic, T. C. Green, Modeling, Analysis and Testing of Autonomous Operation of an Inverter-Based Microgrid, IEEE Transactions on Power Electronics 22 (2) (2007) 613-625.

[5] S. Zuo, A. Davoudi, Y. Song, F. L. Lewis, Distributed finite-time voltage and frequency restoration in islanded AC microgrids, IEEE Transactions on Industrial Electronics 63 (10) (2016) 5988-5997.

[6] N. M. Dehkordi, N. Sadati, M. Hamzeh, Distributed robust finite-time secondary voltage and frequency control of islanded microgrids, IEEE Transactions on Power Systems 32 (5) (2017) 3648-3659.

[7] B. Babaiahgari, M. H. Ullah, J.-D. Park, Coordinated control and dynamic optimization in DC microgrid systems, International Journal of Electrical Power \& Energy Systems 113 (2019) 832-841.
[8] A. B. Siddique, M. S. Munsi, S. K. Sarker, S. K. Das, M. R. Islam, Voltage and current control augmentation of islanded microgrid using multifunction model reference modified adaptive PID controller, International Journal of Electrical Power \& Energy Systems 113 (2019) 492-501.

[9] Y. Guo, Q. Wu, H. Gao, F. Shen, Distributed voltage regulation of smart distribution networks: Consensus-based information synchronization and distributed model predictive control scheme, International Journal of Electrical Power \& Energy Systems 111 (2019) 58-65.

[10] R. Olfati-Saber, R. M. Murray, Consensus problems in networks of agents with switching topology and time-delays, IEEE Transactions on Automatic Control 49 (9) (2004) 1520-1533.

[11] W. Ren, R. W. Beard, Consensus seeking in multiagent systems under dynamically changing interaction topologies, IEEE Transactions on $\mathrm{Au}-$ tomatic Control 50 (5) (2005) 655-661.

[12] R. Olfati-Saber, J. A. Fax, R. M. Murray, Consensus and Cooperation in Networked Multi-Agent Systems, Proceedings of the IEEE 95 (1) (2007) 215-233.

[13] W. Ren, R. W. Beard, E. M. Atkins, Information consensus in multivehicle cooperative control, IEEE Control Systems Magazine 27 (2) (2007) 71-82.

[14] N. Gaeini, A. M. Amani, M. Jalili, X. Yu, Cooperative secondary frequency control of distributed generation: The role of data communication network topology, International Journal of Electrical Power \& Energy Systems 92 (2017) 221-229.

[15] T. Morstyn, B. Hredzak, V. G. Agelidis, Distributed cooperative control of microgrid storage, IEEE Transactions on Power Systems 30 (5) (2015) 2780-2789.

[16] Y. Guo, H. Gao, Q. Wu, Distributed cooperative voltage control of wind 
farms based on consensus protocol, International Journal of Electrical Power \& Energy Systems 104 (2019) 593-602.

17] A. Bidram, A. Davoudi, F. L. Lewis, J. M. Guerrero, Distributed cooperative secondary control of microgrids using feedback linearization, IEEE Transactions on Power Systems 28 (3) (2013) 3462-3470.

[18] A. Bidram, A. Davoudi, F. L. Lewis, Z. Qu, Secondary control of microgrids based on distributed cooperative control of multi-agent systems, IET Generation, Transmission \& Distribution 7 (8) (2013) 822-831.

[19] N. M. Dehkordi, N. Sadati, M. Hamzeh, Fully Distributed Cooperative Secondary Frequency and Voltage Control of Islanded Microgrids, IEEE Transactions on Energy Conversion 32 (2) (2017) 675-685.

[20] J. W. Simpson-Porco, F. Drfler, F. Bullo, Synchronization and power sharing for droop-controlled inverters in islanded microgrids, Automatica 49 (9) (2013) 2603 - 2611.

[21] J. W. Simpson-Porco, Q. Shafiee, F. Drfler, J. C. Vasquez, J. M. Guerrero, F. Bullo, Secondary Frequency and Voltage Control of Islanded Microgrids via Distributed Averaging, IEEE Transactions on Industrial Electronics 62 (11) (2015) 7025-7038.

22] G. Lou, W. Gu, J. Wang, W. Sheng, L. Sun, Optimal Design for Distributed Secondary Voltage Control in Islanded Microgrids: Communication Topology and Controller, IEEE Transactions on Power Systems 34 (2) (2019) 968-981.

[23] W. Levine, M. Athans, On the determination of the optimal constant output feedback gains for linear multivariable systems, IEEE Transactions on Automatic Control 15 (1) (1970) 44-48.

[24] Q. Shafiee, J. M. Guerrero, J. C. Vasquez, Distributed Secondary Contro for Islanded Microgrids-A Novel Approach, IEEE Transactions on Power Electronics 29 (2) (2014) 1018-1031.

[25] J. M. Guerrero, J. C. Vasquez, J. Matas, L. G. de Vicuna, M. Castilla, Hierarchical Control of Droop-Controlled AC and DC MicrogridsA General Approach Toward Standardization, IEEE Transactions on Industrial Electronics 58 (1) (2011) 158-172.

[26] J. M. Guerrero, M. Chandorkar, T. Lee, P. C. Loh, Advanced Control Architectures for Intelligent Microgrids-Part I: Decentralized and Hierarchical Control, IEEE Transactions on Industrial Electronics 60 (4) (2013) 1254-1262.

[27] S. Liu, X. Wang, P. X. Liu, Impact of Communication Delays on Secondary Frequency Control in an Islanded Microgrid, IEEE Transactions on Industrial Electronics 62 (4) (2015) 2021-2031

[28] E. A. A. Coelho, D. Wu, J. M. Guerrero, J. C. Vasquez, T. Dragievi, C. Stefanovi, P. Popovski, Small-Signal Analysis of the Microgrid Secondary Control Considering a Communication Time Delay, IEEE Transactions on Industrial Electronics 63 (10) (2016) 6257-6269.

[29] X. Lu, X. Yu, J. Lai, J. M. Guerrero, H. Zhou, Distributed Secondary Voltage and Frequency Control for Islanded Microgrids With Uncertain Communication Links, IEEE Transactions on Industrial Informatics 13 (2) (2017) 448-460.

[30] Z. Li, Z. Duan, Cooperative control of multi-agent systems: a consensus region approach, CRC Press, 2014.

[31] H. Zhang, F. L. Lewis, Z. Qu, Lyapunov, adaptive, and optimal design techniques for cooperative systems on directed communication graphs, IEEE Transactions on Industrial Electronics 59 (7) (2012) 3026-3041.

[32] J.-J. E. Slotine, W. Li, Applied nonlinear control, Prentice-Hall, Inc., Englewood Cliffs, New Jersey, USA, 1991.

[33] T. Sadamoto, A. Chakrabortty, T. Ishizaki, J. Imura, Dynamic Modeling, Stability, and Control of Power Systems With Distributed Energy Resources: Handling Faults Using Two Control Methods in Tandem, IEEE Control Systems Magazine 39 (2) (2019) 34-65.

[34] H. K. Khalil, Nonlinear Systems, Prentice-Hall, Upper Saddle River, New Jersey, USA, 3 edn., 2002

[35] Z. Li, Z. Duan, F. L. Lewis, Distributed robust consensus control of multiagent systems with heterogeneous matching uncertainties, Automatica 50 (3) (2014) 883-889.

[36] Y. Hua, X. Dong, Q. Li, Z. Ren, Distributed Time-Varying Formation Robust Tracking for General Linear Multiagent Systems With Parameter Uncertainties and External Disturbances, IEEE Transactions on Cybernetics 47 (8) (2017) 1959-1969.

[37] Y. Lv, Z. Li, Z. Duan, G. Feng, Novel distributed robust adaptive consensus protocols for linear multi-agent systems with directed graphs and external disturbances, International Journal of Control 90 (2) (2017) 137147.
[38] R. Christie, IEEE 14-Bus System URL https://item.bettergrids. org/handle/1001/237

[39] J. Stoustrup, A. Annaswamy, A. Chakrabortty, Z. Qu, Smart Grid Control: Overview and Research Opportunities, Springer Nature, Switzerland AG, 1 edn., 2019. 